\title{
HARDY SPACES OF VECTOR-VALUED FUNCTIONS: DUALITY
}

\author{
OSCAR BLASCO
}

Dedicated to the memory of J. L. Rubio de Francia

\begin{abstract}
We prove here that the Hardy space of $B$-valued functions $H^{1}(B)$ defined by using the conjugate function and the one defined in terms of $B$ valued atoms do not coincide for a general Banach space. The condition for them to coincide is the UMD property on $B$. We also characterize the dual space of both spaces, the first one by using $B^{*}$-valued distributions and the second one in terms of a new space of vector-valued measures, denoted $\mathscr{B} \mathscr{O} O\left(B^{*}\right)$, which coincides with the classical $\operatorname{BMO}\left(B^{*}\right)$ of functions when $B^{*}$ has the RNP.
\end{abstract}

Introduction. When the theory of Hardy spaces began to be studied by using the so-called real techniques, several characterizations for these spaces were obtained. We shall consider Hardy spaces on the circle $\mathbf{T}$. Let us write three equivalent formulations of $H^{1}$.

(1) $H_{\text {con }}^{1}=\left\{f \in L^{1}(\mathbf{T}): \tilde{f} \in L^{1}(\mathbf{T})\right\}$,

(2) $H_{\max }^{1}=\left\{f \in L^{1}(\mathbf{T}): P^{*} f(t)=\sup _{0<r<1}\left|P_{r} * f(t)\right| \in L^{1}(\mathbf{T})\right\}$,

(3) $H_{\text {at }}^{1}=\left\{f \in L^{1}(\mathbf{T}): f=\sum \lambda_{k} a_{k}, \sum\left|\lambda_{k}\right|<\infty, a_{k}\right.$ atom $\}$,

where $\tilde{f}$ stands for the conjugate function of $f$ and $P_{r}$ for the Poisson kernel on the circle $\mathbf{T}$.

In 1971, D. L. Burkholder, R. F. Gundy and M. L. Silverstein [7] connected the spaces defined by the conjugate function (1) and by the radial maximal function (2) showing that $H_{\text {con }}^{1}=H_{\max }^{1}$ with equivalent norms. Later, R. R. Coifman [10] gave a constructive proof of the so-called atomic decomposition of functions in $H_{\max }^{1}$, stating that $H_{\max }^{1}=H_{\mathrm{at}}^{1}$ with equivalent norms. In this paper we shall prove that in the vector-valued case Burkholder-Gundy-Silverstein's result holds only for special kinds of Banach spaces. One of the most famous results in Hardy spaces theory was obtained by C. Fefferman (see $[\mathbf{1 5}, \mathbf{1 6}]$ ) by proving that the dual space of $H_{\text {con }}^{1}$ could be identified with the BMO space (functions of bounded mean oscillation) defined by F. John and L. Nirenberg [19]. This duality result leads immediately to the atomic decomposition for functions in $H_{\text {con }}^{1}$. A direct proof of the duality $\left(H_{\mathrm{at}}^{1}\right)^{*}=\mathrm{BMO}$ can be found in [11 or 20].

The aim of this paper is to consider the above spaces when the functions are allowed to take values in a general Banach space $B$, to study the relationship between them and to characterize their dual spaces. The paper is divided into three

Received by the editors October 29, 1986.

1980 Mathematics Subject Classification (1985 Revision). Primary 46E30; $28 \mathrm{~B} 05$.

Key words and phrases. Bounded mean oscillation measures, UMD spaces, Radon-Nikodym property, vector-valued atoms.

Supported by Grant PB85-0338, C.A.I.C.Y.T. 
sections. The first one shows that $H_{\text {con }}^{1}(B)$ and $H_{\max }^{1}(B)$, defined in the obvious way, need not be the same. The space $H_{\text {con }}^{1}(B)$ is always included in $H_{\max }^{1}(B)$, being the necessary and sufficient condition for them to coincide with the UMD property. The second part is devoted to giving a representation of the dual space of $H_{\text {con }}^{1}(B)$ in terms of $B^{*}$-valued distributions. Finally in the last section we define a new space of vector-valued measures, denoted by $\mathscr{B} \mathscr{M} \mathscr{O}(B)$, so that we can write the general duality result $\left(H_{\mathrm{at}}^{1}(B)\right)^{*}=\mathscr{B} \mathscr{M} \mathscr{O}\left(B^{*}\right)$. Besides we find the RadonNikodym property on $B$ as the right one to make the space of measures $\mathscr{B} \mathscr{M} \mathscr{O}(B)$ coincide with the classical space of functions $\mathrm{BMO}(B)$.

Throughout this paper $(B,\|\|)$ will denote a real Banach space, $L^{p}(B)$ will stand for the $B$-valued measurable functions on $\mathbf{T}$ such that

$$
\|f\|_{p}=\left(\frac{1}{2 \pi} \int_{0}^{2 \pi}\|f(t)\|^{p} d t\right)^{1 / p} .
$$

1. Two different Hardy spaces. We can replace the absolute value in Definitions (2) and (3) by the norm of $B$ to get the corresponding $B$-valued Hardy spaces

$$
\begin{gathered}
H_{\max }^{1}(B)=\left\{f \in L^{1}(B): P^{*} f(t)=\sup _{0<r<1}\left\|P_{r^{*}} f(t)\right\| \in L^{1}\right\}, \\
H_{\mathrm{at}}^{1}(B)=\left\{f \in L^{1}(B): f=\sum \lambda_{k} a_{k}, \sum\left|\lambda_{k}\right|<\infty, a_{k} B \text {-atom }\right\} .
\end{gathered}
$$

We define in these the following norms

$$
\begin{gathered}
\|f\|_{\max }=\left\|P^{*} f\right\|_{1}, \\
\|f\|_{\text {at }}=\inf \left\{\sum\left|\lambda_{k}\right|: f=\sum \lambda_{k} a_{k}\right\} .
\end{gathered}
$$

The reader is referred to $[\mathbf{1 0}, \mathbf{1 4}, \mathbf{1 7}]$ for terminology and concepts used in the definitions.

The first result we would like to mention here is that Coifman's proof [10] can be merely reproduced in the $B$-valued setting and consequently we can write

$$
H_{\max }^{1}(B)=H_{\mathrm{at}}^{1}(B) \quad \text { with equivalent norms. }
$$

The definition of $H_{\text {con }}^{1}(B)$ needs a slight remark. Since the existence of the conjugate function cannot be guaranteed (take $B=l^{1}$ to see where the problem arises), we have to assume the existence of the conjugate function and also that this belongs to $L^{1}(B)$, that is

$$
H_{\text {con }}^{1}(B)=\left\{f \in L^{1}(B): \tilde{f} \in L^{1}(B)\right\} .
$$

An easy way of looking at $\tilde{f}$ is as the following limit:

$$
\tilde{f}(t)=\lim _{r 1} Q_{r} * f(t) \quad t \text {-a.e. }
$$

where $Q_{r}$ stands for the conjugate Poisson kernel.

The norm on this last space is given by

$$
\|f\|_{\text {con }}=\|f\|_{1}+\|\tilde{f}\|_{1} .
$$

Our next objective is to show that $H_{\text {con }}^{1}(B) \subset H_{\max }^{1}(B)$ for a general Banach space. To do this we shall use the following lemma whose proof follows easily from the one of the real-valued case (see $[\mathbf{4}, \mathbf{1 8}]$ ). 
LEMMA 1.1. Let $B_{0}$ be a complex Banach space, $D$ the unit disc, and $F$ a holomorphic function on the disc with values in $B_{0}$ belonging to $H^{1}\left(D, B_{0}\right)$, that is

$$
\|F\|_{H^{1}}=\sup _{0<r<1} \frac{1}{2 \pi} \int_{0}^{2 \pi}\left\|F\left(r e^{i t}\right)\right\|_{B_{0}} d t<\infty .
$$

Then

$$
\frac{1}{2 \pi} \int_{0}^{2 \pi} \sup _{0<r<1}\left\|F\left(r e^{i t}\right)\right\|_{B_{0}} d t \leq C\|F\|_{H^{1}}
$$

THEOREM 1.1. $H_{\text {con }}^{1}(B)$ is continuously embedded in $H_{\max }^{1}(B)$.

ProOF. Take $f$ in $H_{\text {con }}^{1}(B)$ and consider $B_{0}=B+i B$ with the norm $\|a+i b\|=$ $\|a\|+\|b\|$. Let us define the following function

$$
F\left(r e^{i t}\right)=P_{r} * f(t)+i P_{r} * f(t)
$$

It is very easy to verify that $F$ belongs to $H^{1}\left(D, B_{0}\right)$ and therefore applying Lemma 1.1, we get

$$
\frac{1}{2 \pi} \int_{0}^{2 \pi} \sup _{0<r<1}\left\|P_{r} * f(t)\right\| d t \leq \frac{1}{2 \pi} \int_{0}^{2 \pi} \sup _{0<r<1}\left\|F\left(r e^{i t}\right)\right\|_{B_{0}} d t \leq\|f\|_{\text {con }},
$$

which finishes the proof.

Let us observe that this result, according to (1.3), is a different approach to the fact, proved by Bourgain [4] using Brownian motion, that every function $f$ in $L^{1}(B)$ with $\hat{f}(n)=0$ for $n<0$ can be decomposed into atoms.

Out next goal is to characterize the class of spaces $B$ where $H_{\text {con }}^{1}(B)$ coincides with $H_{\max }^{1}(B)$, i.e. Burkholder-Gundy-Silverstein's result remains valid. These spaces will be those where the martingale differences are unconditional, called UMD spaces. We shall introduce this property by using a very well-known result due to Bourgain [3], MacConnell and Burkholder [6] which characterized the UMD property in terms of the boundedness of the conjugate function for functions in $L^{p}(B)$.

DEFINITION 1.1. A Banach space $B$ is said to be a UMD space, or to have the UMD property, if there exist a value of $p, 1<p<\infty$, and a constant $C_{p}$ such that

$$
\|\tilde{f}\|_{p} \leq C_{p}\|f\|_{p} \quad \text { for all } f \in L^{p}(B)
$$

Standard techniques show that instead of taking $L^{p}$ norm we can work with $L \log L^{+}(B)$, and then we can state the following theorem.

THEOREM A [21]. Let $1<p<\infty$. The following statements are equivalent.

(a) $B$ is a UMD space.

(b) There are constants $C$ and $C^{\prime}$ such that

$$
\|f\|_{1} \leq C\|f\|_{L \log L^{+}}+C^{\prime} .
$$

The direct implication of the next theorem can be obtained from results in $[23,6$, and 4]. Here we present a proof for the sake of completeness. 
THEOREM 1.2. The following statements are equivalent.

(a) $H_{\text {con }}^{1}(B)=H_{\max }^{1}(B)$ with equivalent norms.

(b) $B$ is an UMD space.

ProOF. Assume that every function in $H_{\max }^{1}(B)$ belongs to $H_{\text {con }}^{1}(B)$, and take a function $f$ in $L \log ^{+} L(B)$. Due to the simple fact that

$$
P^{*} f(t)=\sup _{0<r<1}\left\|P_{r} * f(t)\right\| \leq \sup _{0<r<1} P_{r} *\|f\|(t) \leq M(\|f\|)(t)
$$

where $M f$ stands for the Hardy-Littlewood maximal function, observe that since $\|f\| \in L \log ^{+} L(\mathbf{T})$ then $M(\|f\|) \in L^{1}(\mathbf{T})$, which implies that $f$ belongs to $H_{\max }^{1}(B)$, and therefore, by assumption, to $H_{\text {con }}^{1}(B)$. Moreover we have

$$
\|f\|_{1} \leq C\|f\|_{L \log L^{+}}+C \text {. }
$$

Conversely, according to (1.3), we shall prove that there is a constant $C$ such that for all $B$-atoms $a$ it verifies

$$
\|\tilde{a}\|_{1} \leq C .
$$

The first observation is that the conjugate function of an atom exists since we are assuming $B$ is UMD, and $a \in L^{2}(B)$. Assume $a$ is supported in $(-\delta, \delta)$ for some $\delta>0$ (here we are identifying $\mathbf{T}$ with $[-\pi, \pi)$ ).

$$
\int_{-2 \delta}^{2 \delta}\|a(t)\| d t \leq\left(\int_{-2 \delta}^{2 \delta}\|a(t)\|^{2} d t\right)^{1 / 2}(2 \delta)^{1 / 2} \leq C\|a\|_{2} \delta^{1 / 2} \leq C .
$$

To check the integral over $\{t: 2 \delta<|t|<\pi\}$ we use the fact that

$$
\int_{2 \delta<|t|<\pi}\left|Q_{r}(t-s)-Q_{r}(t)\right| d t \leq C \quad \text { for all } r, 0<r<1
$$

and a standard computation with atoms shows that

$$
\begin{gathered}
\int_{2 \delta<|t|<\pi}\left\|Q_{r} * a(t)\right\| d t=\int_{2 \delta<|t|<\pi}\left\|\int_{-\delta}^{\delta}\left(Q_{r}(t-s) a(s)-Q_{r}(t) a(s)\right) d s\right\| d t \\
\leq \int_{-\delta}^{\delta}\left(\int_{2 \delta<|t|<\pi}\left|Q_{r}(t-s)-Q_{r}(t)\right| d t\right)\|a(s)\| d s \leq C\|a\|_{1} \leq C .
\end{gathered}
$$

From this last inequality we get

$$
\int_{2 \delta<|t|<\pi}\|\tilde{a}(t)\| d t \leq C .
$$

Once we have proved (1.8) we can extend this to any function $f$ in $H_{\mathrm{an}}^{1}(B)$ and easily show that

$$
\|\tilde{f}\|_{1} \leq C\|f\|_{1} \quad \text { for } f \text { in } H_{\mathrm{at}}^{1}(B),
$$

which implies, using (1.3), that $H_{\max }^{1}(B) \subset H_{\text {con }}^{1}(B)$, and since the other inclusion was proved in Theorem 1.1, then the proof is completed.

REMARK 1.1. There is another very interesting space to consider in the setting of vector-valued Hardy spaces: $H_{\text {con }}^{1} \hat{\otimes} B$, that is the tensor product with the projective norm. Several results about it can be seen in [18]. Here we only want to mention that in general $H_{\text {con }}^{1} \hat{\otimes} B \subset H_{\text {con }}^{1}(B)$. The following example gives equality $B=l^{1}$. To see this it is enough to realize that $H_{\text {con }}^{1}\left(l^{1}\right)$ can be interpreted as $l^{1}\left(H^{1}\right)$ and then use the very well-known fact that $l^{1} \hat{\otimes} B=l^{1}(B)$ for any Banach space $B$. 
2. Duality for $H_{\text {con }}^{1}(B)$. To characterize the dual space of $H_{\text {con }}^{1}(B)$ we shall introduce the concepts of $B$-valued distribution and conjugate distribution.

Let us denote $C^{\infty}(\mathbf{T})$ the space of $2 \pi$-periodic functions in $C^{\infty}(\mathbf{R})$ endowed with the topology given by the seminorms $\left(p_{m}: m \in \mathbf{N}\right)$ where

$$
p_{m}(\phi)=\sup _{0 \leq k \leq m} \sup _{t \in \mathbf{T}}\left|\phi^{k}(t)\right| \text {. }
$$

DEFinition 2.1. The continuous linear maps from $C^{\infty}(\mathbf{T})$ into $B$ will be called $B$-valued distributions and the space will be denoted by $\mathscr{D}^{\prime}(B)$. The main point to consider in this space is given by the following fact:

$$
\text { If } \varphi \in C^{\infty}(\mathbf{T}) \text { then } \tilde{\varphi} \in C^{\infty}(\mathbf{T}) \text {. }
$$

This enables us to define the conjugate of a distribution $T$ in $\mathscr{D}^{\prime}(B)$ as the $B$-valued distribution given by

$$
\tilde{T}(\varphi)=-T(\tilde{\varphi}) \quad \text { for all } \varphi \text { in } C^{\infty}(\mathbf{T}) .
$$

Given an element $\Phi$ in $\left(H_{\text {con }}^{1}(B)\right)^{*}$ we can define the following $B^{*}$-valued distribution

$$
\left\langle T_{\Phi}(\psi), b\right\rangle=\Phi(\psi \cdot b) \quad \text { for all } \psi \in C^{\infty}(\mathbf{T}), b \in B
$$

where $\psi \cdot b$ denotes the $B$-valued function $(\psi \cdot b)(t)=\psi(t) \cdot b$.

Under this identification we are able to look at the dual space of $H_{\text {con }}^{1}(B)$ as certain class distributions in $\mathscr{D}^{\prime}\left(B^{*}\right)$. We shall denote by $\Lambda^{\infty}(B)$ the class of distributions which can be extended to elements in $\mathscr{L}\left(L^{1}(\mathbf{T}), B\right)$, that is, verifying that there exists a constant $C$ such that

$$
\|T(\psi)\| \leq C\|\psi\|_{1} \quad \text { for all } \psi \text { in } C^{\infty}(\mathbf{T}),
$$

where the norm in it is given by the infimum of the constant verifying (2.4).

Observe that

$$
\Lambda^{\infty}\left(B^{*}\right)=\mathscr{L}\left(L^{1}(\mathbf{T}), B^{*}\right)=\left(L^{1} \hat{\otimes} B\right)^{*}=\left(L^{1}(B)\right)^{*} .
$$

Finally let us denote by $\tilde{\Lambda}^{\infty}(B)=\left\{T \in \mathscr{D}^{\prime}(B): \widetilde{T} \in \Lambda^{\infty}(B)\right\}$ where $\|T\|_{\tilde{\Lambda}^{\infty}}=\|\tilde{T}\|$.

THEOREM 2.1. $\left(H_{\text {con }}^{1}(B)\right)^{*}=\Lambda^{\infty}\left(B^{*}\right)+\tilde{\Lambda}^{\infty}\left(B^{*}\right)$.

Proof. Let $\Phi$ be an element in $\left(H_{\text {con }}^{1}(B)\right)^{*}$. Since the application $f \rightarrow(f, \tilde{f})$ maps $H_{\text {con }}^{1}(B)$ into $L^{1}(B) \oplus L^{1}(B)$ then the Hahn-Banach extension theorem allows us to find $\Phi_{1}, \Phi_{2}$ belonging to $\left(L^{1}(B)\right)^{*}$ such that

$$
\Phi(f)=\Phi_{1}(f)+\Phi_{2}(\tilde{f}) \quad \text { for all } f \text { in } H_{\text {con }}^{1}(B) .
$$

By the last remark we can consider $T_{1}, T_{2}$, in $\Lambda^{\infty}\left(B^{*}\right)$ as the corresponding elements for $\Phi_{1}, \Phi_{2}$. We shall prove that $T=T_{1}-\widetilde{T}_{2}$.

$$
\begin{aligned}
\left\langle T_{\Phi}(\psi), b\right\rangle & =\Phi(\psi \cdot b)=\Phi_{1}(\psi \cdot b)+\Phi_{2}(\widetilde{\psi \cdot b}) \\
& =\left\langle T_{1}(\psi), b\right\rangle+\left\langle T_{2}(\tilde{\psi}), b\right\rangle=\left\langle\left(T_{1}-\widetilde{T}_{2}\right)(\psi), b\right\rangle
\end{aligned}
$$

Conversely suppose we take $T=T_{1}+T_{2}$ with $T_{1}, \widetilde{T}_{2}$ belonging to $\Lambda^{\infty}\left(B^{*}\right)$ whose corresponding elements in $\left(L^{1}(B)\right)^{*}$ are $\Phi_{1}, \Phi_{2}$. 
Define $\Phi(f)=\Phi_{1}(f)-\Phi_{2}(\tilde{f})$ for each $f$ in $H_{\text {con }}^{1}(B)$. This is obviously linear and the continuity follows from the following easy inequality

$$
|\Phi(f)| \leq\left|\Phi_{1}(f)\right|+\left|\Phi_{2}(\tilde{f})\right| \leq \max \left(\left\|\Phi_{1}\right\|,\left\|\Phi_{2}\right\|\right)\|f\|_{\text {con }} .
$$

Since it can be shown $T_{\Phi}=T_{1}+T_{2}$ we have finished the proof.

REMARK 1.2. Duality results for Hardy spaces of vector-valued functions have been considered by several authors (see $[\mathbf{4}, \mathbf{5}, \mathbf{2 5}])$. The following result can be stated for special kinds of Banach spaces (see $[\mathbf{5}, \mathbf{2 5}]$ ):

$$
\left(H_{\text {con }}^{p}(B)\right)^{*}=H_{\text {con }}^{p}\left(B^{*}\right) \quad \text { if and only if } B \text { is a UMD space, }(1<p<\infty) \text {. }
$$

Here we shall formulate a duality result for a general Banach space. Let us define the class of distributions in $\mathscr{D}^{\prime}(B)$ such that there exists a positive function $g$ in $L^{p}(\mathbf{T})$ verifying

$$
\|T(\psi)\| \leq\langle g,|\psi|\rangle \quad \text { for all } \psi \in C^{\infty}(\mathbf{T}) .
$$

This space can be identified with the space of cone absolutely summing operators from $L^{p}$ into $B$ (see [26, p. 244]). Denoting this space by $\Lambda^{p}(B)$, we can identify the dual of $L^{p}(B)$ with $\Lambda^{p}\left(B^{*}\right)$ (see [26, p. 277]). Due to this identification a proof such as for Theorem 2.1 would allow us to show the following.

Corollary 2.1. For $1<p<\infty,\left(H_{\text {con }}^{p}(B)\right)^{*} \Lambda^{p^{\prime}}\left(B^{*}\right)+\widetilde{\Lambda}^{p^{\prime}}\left(B^{*}\right)$.

REMARK 2.2. Theorem 2.1 can be also used to give a sufficient condition on $B$ to get $H_{\text {con }}^{1}(B)=H_{\text {con }}^{1} \hat{\otimes} B$. By using the density of $H_{\text {con }}^{1} \hat{\otimes} B$ in $H_{\text {con }}^{1}(B)$ (a fact which can be seen by showing that $\sigma_{n} * f$ converges to $f$ in $H_{\text {con }}^{1}(B), \sigma_{n}$ being the Féjer kernel [21]) we have only to verify that $\left(H_{\text {con }}^{1} \hat{\otimes} B\right)^{*}$ coincides with $\left(H_{\text {con }}^{1}(B)\right)^{*}$. In Theorem 2.1 we have identified the dual of the second one, but the dual of a tensor product is known to be identified with $\mathscr{L}\left(H_{\text {con }}^{1}, B^{*}\right)$ (see [12, p. 230]), and from these two facts is very easy to find a condition to get the equality between both spaces.

Let us recall that a Banach space $X$ is said to have the Hahn-Banach extension property if for every $Y_{1}$ and $Y_{2}$ Banach spaces, $\Omega: Y_{1} \rightarrow Y_{2}$ isometric inclusion and $T$ in $\mathscr{L}\left(Y_{1}, X\right)$ then $T$ can be extended to $\bar{T}$ in $\mathscr{L}\left(Y_{2}, X\right)$ with $\bar{T} \cdot \Omega=T$ and $\|\bar{T}\| \leq\|T\|$.

Now considering the embedding from $H_{\text {con }}^{1}$ into $L^{1} \oplus L^{1}$ given by $\psi \rightarrow(\psi, \tilde{\psi})$ we can easily prove the next result.

COROLlaRY 2.2. If $B^{*}$ has the Hahn-Banach extension property then $H_{\mathrm{con}}^{1}(B)$ $=H_{\text {con }}^{1} \hat{\otimes} B$.

This result has also been pointed out in [18], and proves that the example in Remark 1.1 is not a coincidence since $\left(l^{1}\right)^{*}=l^{\infty}$ has this property.

3. Duality for $H_{\mathrm{at}}^{1}(B)$. In this section we shall give a representation of the dual space of $H_{\mathrm{at}}^{1}(B)$. To do this the first thing to realize is the following inclusions:

$$
L^{p}(B) \subset H_{\mathrm{at}}^{1}(B) \subset L^{1}(B) \quad \text { (with continuity), } \quad 1<p<\infty .
$$

Since each space is dense in the next one, we can also write

$$
\Lambda^{\infty}\left(B^{*}\right)=\left(L^{1}(B)\right)^{*} \subset\left(H_{\mathrm{at}}^{1}(B)\right)^{*} \subset\left(L^{p}(B)\right)^{*}=\Lambda^{p^{\prime}}\left(B^{*}\right) .
$$


Instead of looking at $\Lambda^{\infty}\left(B^{*}\right)$ and $\Lambda^{p^{\prime}}\left(B^{*}\right)$ as spaces of distributions, or equivalently of operators in $\mathscr{L}\left(L^{1}, B^{*}\right)$ and $\mathscr{L}\left(L^{p}, B^{*}\right)$, we shall regard them as spaces of $B^{*}$ valued measures. The identification is quite obvious:

Given $T$ in $\mathscr{L}\left(L^{p}, B\right)$ we define the measure

$$
G(E)=T\left(\chi_{E}\right) \quad \text { for all measurable sets } E .
$$

The spaces $\Lambda^{p}(B)$ will correspond to certain classes of $B$-valued measures defined as follows

DEFINITION 3.1. Let $1<p<\infty$. Let $G$ be a $B$-valued infinitely additive measure on $(\mathbf{T}, \mathscr{B})$. $G$ is said to have bounded $p$-variation if

$$
|G|_{p}=\sup \left(\sum_{E \in \Pi} \frac{\|G(E)\|^{p}}{m(E)^{p-1}}\right)^{1 / p}<\infty
$$

(where the supremum is taken over all finite partitions of $\mathbf{T}$ and where $\lambda / 0$ is interpreted by 0 if $\lambda=0$ or by $+\infty$ if $\lambda>0$ ). $G$ is said to have $\infty$-bounded variation if

$$
|G|_{\infty}=\inf \{C:\|G(E)\| \leq C m(E) \text { for all } E\}<+\infty .
$$

We shall denote by $V^{p}(B)$ the space of measures of bounded $p$-variation $(1<$ $p \leq \infty)$. These spaces have been connected with spaces of operators in several ways (see $[13,2])$.

Before we define the spaces of vector-valued measures we shall work with, let us introduce some other notation. Given a measurable set $E$ and a measure $G$, we shall denote by $G_{E}$ the $B$-valued measure restricted to $\left(E, \mathscr{B}_{E}, m_{E}\right)$ where $\mathscr{B}_{E}=\{A \cap E: A \cap \mathscr{B}\}$ and $m_{E}(A)=m(A \cap E)$. According to the definition of 2-variation we can write

$$
\left|G_{E}\right|_{2}=\sup \left(\sum_{A \in \Pi_{E}} \frac{\|G(A)\|^{2}}{m(A)}\right)^{1 / 2}
$$

where the sup is taken over all finite partitions $\Pi_{E}$ of $E$.

DEFINITION 3.2. Let $G$ be a finitely additive measure with values in $B$. Let $I$ be an interval, and consider the following measure on $\left(I, \mathscr{B}_{I}, m_{I}\right)$ :

$$
G_{I}^{*}=G_{I}-(G(I) / m(I)) m_{I} .
$$

$G$ is said to belong to $\mathscr{B} \mathscr{M} \mathscr{O}(B)$ if

$$
|G|_{*}=\sup \left(M(I)^{-1 / 2}\left|G_{I}^{*}\right|_{2}: I \text { interval }\right)<\infty .
$$

Observe that $|G|_{*}=0$ does not imply $G=0$ (since $G(E)=b m(E)$ for a constant vector $b$ satisfies $|G|_{*}=0$ ). Therefore we shall introduce the following norm in $\mathscr{B} \mathscr{M} \odot(B)$ :

$$
\|G\|_{\mathrm{BMO}}=|G|_{*}+\|G(\mathbf{T})\| .
$$

An equivalent useful norm can be given by replacing $|G|_{*}$ by

$$
|G|_{*}^{\prime}=\sup \left\{\inf _{b \in B} m(I)^{-1 / 2}\left|G_{I}-b m_{I}\right|_{2}: I \text { interval }\right\} \text {. }
$$


Proposition 3.1. Let $G$ be a finitely additive measure, then $|G|_{*}^{\prime} \leq|G|_{*} \leq$ $2|G|_{*}$.

PROOF. The first inequality is completely obvious. To see the converse let us take a vector $b$ in $B$ and an interval $I$. Then we can write

$$
G_{I}^{*}=G_{I}-b m_{I}-(G(I) / m(I)-b) m_{I} .
$$

Since $V^{2}(B)$ is a normed space we have

$$
\left|G_{I}^{*}\right|_{2} \leq\left|G_{I}-b m_{I}\right|_{2}+\left|(G(I) / m(I)-b) m_{I}\right|_{2} .
$$

Taking a look at (3.4) and considering the particular partition given only by $I$ we get

$$
\|G(I)-b m(I)\| \leq\left|G_{I}-b m_{I}\right|_{2} m(I)^{1 / 2} .
$$

Therefore the result follows from this and the fact $\left|m_{I}\right|_{2}=m(I)^{1 / 2}$.

PROPOSITION 3.2. $V^{\infty}(B) \subset \mathscr{B} \mathscr{M} \mathscr{O}(B) \subset V^{2}(B)$ (with continuity).

ProOF. Let $G$ be a measure in $V^{\infty}(B)$. Then $\left\|G_{I}^{*}(A)\right\| \leq 2|G|_{\infty} m(A)$. This easily implies that $|G|_{*} \leq 2|G|_{\infty}$. Assume now that $G$ belongs to $\mathscr{B} \mathscr{M} \mathscr{O}(B)$, and take $I=\mathbf{T}$. Then we have $G_{I}^{*}=G-G(\mathbf{T}) m$ and $|G|_{2} \leq\left|G^{*}\right|_{2}+\|G(\mathbf{T})\| \leq$ $\|G\|_{\text {BMO. }}$

Because of this last proposition and the good properties of measures in $V^{2}(B)$ (see $[\mathbf{1 3}]$ ) we can write

COROLlaRY 3.1. If $G$ belongs to $\mathscr{B} \mathscr{M} O(B)$ then $G$ is countable additive, $m$ continuous and with bounded variation.

The following result connects the space of functions in $\operatorname{BMO}(B)$, whose definition is like the classical one replacing the absolute value by the norm (see $[\mathbf{1 7}, 19$ and 11] for the definitions and previous properties) with the space of measures.

PROPOSITION 3.3. Let $f$ belong to $L^{1}(B)$ and consider $G(E)=\int_{E} f(t) d m(t)$. $G \in \mathscr{B} \mathscr{M}(B)$ if and only if $f \in \operatorname{BMO}(B)$.

PROOF. This is simply based on the following result for measures in $V^{2}(B)$ : $G(E)=\int_{E} g(t) d m(t) \in V^{2}(B)$ then $|G|_{2}=\left(\int\|g(t)\|^{2} d m(t)\right)^{1 / 2}$ (see $[\mathbf{2}, \mathbf{1 3}]$ ). Since

$$
G_{I}^{*}(E)=\int_{E}\left(f(t)-f_{I}\right) d m(t) \quad \text { for all } E \text { in } \mathscr{B}_{I}
$$

where $f_{I}=m(I)^{-1} \int_{I} f(t) d m(t)$, then obviously $G$ belonging to $\mathscr{B} \mathscr{M} \mathscr{O}(B)$, together with John-Nirenberg's lemma, is equivalent to $f$ belonging to $\operatorname{BMO}(B)$.

The last proposition implies that $\operatorname{BMO}(B) \subset \mathscr{B} \mathscr{M} \mathscr{O}(B)$ being this is an isometric inclusion. The next one characterizes when both spaces coincide.

PROPOSITION 3.4. $\mathrm{BMO}(B)=\mathscr{B} \mathscr{M} \mathscr{O}(B)$ if and only if $B$ has the RadonNikodym property.

PROOF. A formulation of RNP is that $V^{\infty}(B)=L^{\infty}(B)$ (see [12, p. 63]), so assuming $\operatorname{BMO}(B)=\mathscr{B} \mathscr{M O}(B)$ and taking $G$ in $V^{\infty}(B)$ we deduce that we can represent $G$ by a function $f$ in $L^{1}(B)$. Now a standard argument (see [12, p. 62]) shows that in fact $f$ belongs to $L^{\infty}(B)$. Conversely when we assume $B$ has RNP and we take $G$ in $\mathscr{B} \mathscr{M} \mathscr{O}(B)$, then Corollary 3.1 and Proposition 3.3 finish the proof. 
LEMMA 3.1. Let $G$ be a measure in $\mathscr{B} \mathscr{M}(B)$. Then for each positive interger $n$ there is a simple function $f_{n}$ such that

$$
\left\|f_{n}\right\|_{\mathrm{BMO}} \leq 4\|G\|_{\mathrm{BMO}} \text {. }
$$

(3.10) Denoting by $G_{n}(E)=\int_{E} f_{n}(t) d m(t)$, then $G_{n} \in V^{\infty}(B)$ and for all measurable sets $E, G_{n}(E)$ converges to $G(E)$ as $n$ goes to $\infty$.

PROOF. Denote by $I_{n, k}$ the dyadic interval $\left[k 2^{-n},(k+1) 2^{-n}\right)$ and write $x_{k}=$ $G\left(I_{n, k}\right) / m\left(I_{n, k}\right)$. Let us define the following simple function

$$
f_{n}=\sum_{k=0}^{2^{n}-1} x_{k} \chi_{I_{n, k}}
$$

We shall check

$$
\left|f_{n}\right|_{*}^{\prime}=\sup \left\{\inf _{b \in B}\left(m(I)^{-1} \int_{I}\left\|f_{n}(t)-b\right\|^{2} d t\right)^{1 / 2}: I \text { interval }\right\}
$$

by considering two kinds of intervals. Let us start by taking an interval $I$ with $m(I) \leq 2^{-n}$. Here we have two cases to take into account: The interval $I$ is either contained in some $I_{n, k}$ or it intersects two consecutive intervals $I_{n, k}$ and $I_{n, k+1}$ which we denote by $I^{\prime}$ and $I^{\prime \prime}$. By taking $b_{I}=\left(f_{n}\right)_{I}$, that is the average of $f_{n}$ over the interval $I$, we shall have

$$
m(I)^{-1} \int_{I}\left\|f_{n}(t)-b_{I}\right\|^{2} d t=0
$$

in the first case, and therefore

$$
\inf _{b \in B}\left(m(I)^{-1} \int_{I}\left\|f_{n}(t)-b\right\|^{2} d t\right)^{1 / 2}=0 .
$$

In the second case we shall have

$$
\left(f_{n}\right)_{I}=x_{I^{\prime}} \frac{m\left(I \cap I^{\prime}\right)}{m(I)}+x_{I^{\prime \prime}} \frac{m\left(I \cap I^{\prime \prime}\right)}{m(I)}
$$

and then

$$
\begin{aligned}
& m(I)^{-1} \int_{I}\left\|f_{n}(t)-b_{I}\right\|^{2} d t \\
& \quad=m(I)^{-1}\left(\left\|x_{I^{\prime}}-b_{I}\right\|^{2} m\left(I \cap I^{\prime}\right)+\left\|x_{I^{\prime \prime}}-b_{I}\right\|^{2} m\left(I \cap I^{\prime \prime}\right)\right) .
\end{aligned}
$$

A very easy computation shows that

$$
\begin{aligned}
& \left\|x_{I^{\prime}}-b_{I}\right\|=\left\|x_{I^{\prime}}-x_{I^{\prime \prime}}\right\| m\left(I \cap I^{\prime \prime}\right) m(I)^{-1}, \\
& \left\|x_{I^{\prime \prime}}-b_{I}\right\|=\left\|x_{I^{\prime \prime}}-x_{I^{\prime}}\right\| m\left(I \cap I^{\prime}\right) m(I)^{-1} .
\end{aligned}
$$

To compute $\left\|x_{I^{\prime}}-x_{I^{\prime \prime}}\right\|$ we shall use the triangle inequality and we compute $\left\|x_{I^{\prime}}-x_{I^{\prime} \cup I^{\prime \prime}}\right\|+\left\|x_{I^{\prime \prime}}-x_{I^{\prime} \cup I^{\prime \prime}}\right\|$. Since $I^{\prime}$ and $I^{\prime \prime}$ are consecutive intervals and $I$ is contained in $I^{\prime} \cup I^{\prime \prime}$, being $2 m\left(I^{\prime}\right)=2 m\left(I^{\prime \prime}\right)=m\left(I^{\prime} \cup I^{\prime \prime}\right)$, we can easily show

$$
\left\|x_{I^{\prime}}-x_{I^{\prime \prime} \cup I^{\prime}}\right\| \leq 2|G|_{*} \quad \text { and } \quad\left\|x_{I^{\prime \prime}}-x_{I^{\prime} \cup I^{\prime \prime}}\right\| \leq 2|G|_{*} .
$$


Putting it all together we have

$$
\left(m(I)^{-1} \int_{I}\left\|f_{n}(t)-b_{I}\right\|^{2} d t\right)^{1 / 2} \leq 4|G|_{*},
$$

which shows that for an interval with $m(I) \leq 2^{-n}$ we are done.

Let us consider now an interval $I$ with $m(I)>2^{-n}$, denoting by $I$ the union of dyadic intervals such that $I \subset \bar{I}=\left\{I_{n, k}, k \in F(I)\right\}$ and $I \leq m(\bar{I}) / m(I) \leq 3$. In this case we shall choose $b_{I}=G(\bar{I}) / m(\bar{I})$.

$$
\begin{aligned}
m(I)^{-1} & \int_{I}\left\|f_{n}(t)-b_{I}\right\|^{2} d t \leq 3 m(\bar{I})^{-1} \int_{\bar{I}}\left\|f_{n}(t)-b_{I}\right\|^{2} d t \\
& =3 m(\bar{I})^{-1} \sum_{k \in F(I)}\left\|x_{k}-b_{I}\right\|^{2} m\left(I_{n, k}\right) \\
& \leq 3 m(\bar{I})^{-1}\left|G_{\bar{I}}-b_{I} m_{\bar{I}}\right|_{2}^{2} \leq 3|G|_{*}^{2} .
\end{aligned}
$$

Now joining this last inequality with (3.13) we can get easily (3.9). To obtain (3.10) it suffices to notice that the Borel $\sigma$-algebra $\mathscr{B}$ is generated by $\bigcup_{n} \mathscr{B}_{n}$ where $\mathscr{B}_{n}$ is the algebra given by the dyadic intervals of length $2^{-n}$ and to observe that when we restrict to sets in $\mathscr{B}_{n}$ we have $G_{n}(E)=G(E)$. Finally we have that the fact that $G_{n}$ belongs to $V^{\infty}(B)$ follows from $f_{n}$ being a function in $L^{\infty}(B)$.

Before we state the duality theorem, let us look at the space $H_{a t}^{1}(B)$ in a more convenient way for our purposes.

DEFinition 3.3. A function $a$ in $L^{2}(B)$ is said to be a $(B, 2)$-atom if there exists an interval $I$ such that

(1) $\operatorname{supp} a \subset I$,

(2) $\int_{I} a(t) d m(t)=0$

(3) $\int_{I}\|a(t)\|^{2} d m(t) \leq m(I)^{-1}$.

We also consider $a(t)=b$ for some $b$ in $B$ with $\|b\|=1$ as a $(B, 2)$-atom. A proof such as that given in [11] allows us to consider the space $H_{\mathrm{at}}^{1}(B)$ defined in terms of these atoms. So we consider

$$
H_{\mathrm{at}}^{1}(B)=\left\{f \in L^{1}(B): f=\sum \lambda_{k} a_{k}, a_{k} \text { are }(B, 2) \text {-atoms and } \sum\left|\lambda_{k}\right|<\infty\right\} .
$$

The norm on it is given by

$$
\|f\|_{\text {at }}=\inf \left\{\sum\left|\lambda_{k}\right|: f=\sum \lambda_{k} a_{k}\right\} .
$$

THEOREM 3.1. $\left(H_{\mathrm{at}}^{1}(B)\right)^{*}=\mathscr{B} \mathscr{M} \odot\left(B^{*}\right)$.

ProOF. First let us take $G$ belonging to $\mathscr{B} \mathscr{M} \mathscr{O}\left(B^{*}\right)$. We shall define an operator acting on $B$-valued simple functions and we shall prove that is bounded as an operator acting from the space of simple functions with norm given by $H_{\mathrm{at}}^{1}(B)$ into $\mathbf{R}$, and then we shall extend it to an element in $\left(H_{\mathrm{at}}^{1}(B)\right)^{*}$.

Define the following operator

$$
T_{G}\left(\sum_{k=1}^{n} a_{k} \chi_{E_{k}}\right)=\sum_{k=1}^{n}\left\langle G\left(E_{k}\right), a_{k}\right\rangle .
$$

We shall show that

$$
\left|T_{G}(s)\right| \leq|G|_{\mathrm{BMO}}\|s\|_{\mathrm{at}} \quad \text { for all simple functions } s .
$$


First we use Lemma 3.1 to get a sequence of measures $G_{n}$ in $V^{\infty}\left(B^{*}\right)$. Denote by $T_{n}$ the operator given by (3.15) replacing $G$ by $G_{n}$. The advantage of this one with respect to the first one is that $T_{n}$ defines an element in $\left(L^{1}(B)\right)^{*}$ since $G_{n}$ belongs to $V^{\infty}\left(B^{*}\right)$. Our aim now is to show

$$
\left|T_{n}(F)\right| \leq C\left\|G_{n}\right\|_{\mathrm{BMO}}\|f\|_{\text {at }} \quad \text { for all } f \text { in } H_{\mathrm{at}}^{1}(B) \text {. }
$$

Let us start with a simple atom, that is

$$
s=\sum_{k=1}^{N} a_{k} \chi_{E_{k}}, \quad E_{k} \subset I, \quad \sum_{k=1}^{N} a_{k} m\left(E_{k}\right)=0, \quad \sum_{k=1}^{N}\left\|a_{k}\right\|^{2} m\left(E_{k}\right) \leq 2 m(I)^{-1} .
$$

Denoting $b_{I}=G_{n}(I) / m(I)$ we can write

$$
T_{n}(s)=\sum_{k=1}^{N}\left\langle G_{n}\left(E_{k}\right), a_{k}\right\rangle=\sum_{k=1}^{N}\left\langle G_{n}\left(E_{k}\right)-b_{I} m\left(E_{k}\right), a_{k}\right\rangle .
$$

Therefore

$$
\begin{aligned}
\left|T_{n}(s)\right| & \leq \sum_{k=1}^{N}\left\|G_{n}\left(E_{k}\right)-b_{I} m\left(E_{k}\right)\right\|_{B^{*}} m\left(E_{k}\right)^{-1 / 2} m\left(E_{k}\right)^{1 / 2}\left\|a_{k}\right\| \\
& \leq\left(\sum_{k=1}^{N} \frac{\left\|G_{n}\left(E_{k}\right)-b_{I} m\left(E_{k}\right)\right\|^{2}}{m\left(E_{k}\right)}\right)^{1 / 2} \cdot\left(\sum_{k=1}^{N}\left\|a_{k}\right\|^{2} m\left(E_{k}\right)\right)^{1 / 2} \\
& \leq 2 m(I)^{-1 / 2}\left|\left(G_{n}\right)_{I}-b_{I} m_{I}\right|_{2} \leq 2\left|G_{n}\right|_{*} .
\end{aligned}
$$

Given now a general $(B, 2)$-atom $a$ in $L^{2}(B)$ supported in $I$ we can find a sequence of simple functions $d_{k}$ converging to $a$ in $L^{2}(I, B)$. Taking $s_{k}=d_{k}$ $\left(\int_{I} d_{k}(t) d m(t)\right) \chi_{I}$ we have a sequence of simple atoms which also converges to $a$ in $L^{2}(I, B)$ and therfore in $L^{1}(I, B)$. Using the continuity of $T_{n}$ as an element in $\left(L^{1}(B)\right)^{*}$ we can say

$$
\left|T_{n}(a)\right| \leq 2\left|G_{n}\right|_{*} \quad \text { for nonconstant atoms } a .
$$

For $a(t)=b$ with $\|b\|=1$ we have $\left|T_{n}(a)\right|=\|G(\mathbf{T})\|$, and therefore

$$
\left|T_{n}(a)\right| \leq 2\left\|G_{n}\right\|_{\mathrm{BMO}} \quad \text { for all atoms } a \text {. }
$$

This last inequality, together with the fact that $T_{n} \in\left(L^{1}(B)\right)^{*}$, and the convergence in each representation $f=\sum \lambda_{k} a_{k}$ is also in $L^{1}(B)$, imply (3.17). To finish the proof we invoke Lemma 3.1 which says that $T_{n}(s)$ converges to $T(s)$ for all simple functions $s$ and $\left\|G_{n}\right\|_{\mathrm{BMO}} \leq 4\|G\|_{\mathrm{BMO}}$.

To prove the converse inclusion, let us take $T$ in $\left(H_{\mathrm{at}}^{1}(B)\right)^{*}$, and define the following $B^{*}$-valued measure

$$
\langle G(E), b\rangle=T\left(b \chi_{E}\right) \quad \text { for all } b \in B \text { and } E \in \mathscr{B} \text {. }
$$

Given $n$ intervals $I$ and a partition $\Pi_{I}=\left\{E_{1}, E_{2}, \ldots, E_{n}\right\}$ of $I$, we can write, according to the duality $\left(l^{2}(B)\right)^{*}=l^{2}\left(B^{*}\right)$,

$$
\begin{aligned}
\sum\left\|G\left(E_{i}\right)-G(I) m(I)^{-1} m\left(E_{i}\right)\right\|_{B^{*}}^{2} m\left(E_{i}\right)^{-1} & \\
= & \sum\left\|G\left(E_{i}\right) m\left(E_{i}\right)^{-1 / 2}-G(I) m(I)^{-1} m\left(E_{i}\right)^{1 / 2}\right\|_{B^{*}}^{2} \\
& =\left|\sum\left\langle G\left(E_{i}\right) m\left(E_{i}\right)^{-1 / 2}-G(I) m(I)^{-1} m\left(E_{i}\right)^{1 / 2}, b_{i}\right\rangle\right|^{2} \\
& \text { for some } \sum\left\|b_{i}\right\|^{2}=1 .
\end{aligned}
$$


Therefore using the definition of $G$ we have

$$
\begin{aligned}
& \left|\sum\left(T\left(b_{i} m\left(E_{i}\right)^{-1 / 2} \chi_{E}\right)-T\left(b_{i} m\left(E_{i}\right)^{1 / 2} m(I) \chi_{I}\right)\right)\right| \\
& \quad=\left|T\left(\sum b_{i} m\left(E_{i}\right)^{-1 / 2} \chi_{E}-b m(I)^{-1} \chi_{I}\right)\right|, \quad \text { where } b=\sum m\left(E_{i}\right)^{1 / 2} b_{i} .
\end{aligned}
$$

The reader can easily verify that

$$
a=\frac{1}{2} m(I)^{-1 / 2}\left(\sum m\left(E_{i}\right)^{-1 / 2} b_{i} \chi_{E}-b m(I)^{-1} \chi_{I}\right)
$$

is a $(B, 2)$-atom and therefore we get

$$
\left|G_{I}^{*}\right|_{2} \leq T\left(2 m(I)^{1 / 2} a\right) \mid \leq 2 m(I)^{1 / 2}\|T\| .
$$

Since also $\|G(\mathbf{T})\|_{B^{*}} \leq\|T\|$, then we have $\|G\|_{\mathrm{BMO}} \leq 2\|T\|$ and the proof is finished.

The following result was proved in [1] with a direct proof but now we can get it as a corollary from Theorem 3.1 and Proposition 3.4.

COROLlary 3.2. $\left(H_{\mathrm{at}}^{1}(B)\right)^{*}=\mathrm{BMO}\left(B^{*}\right)$ if and only if $B^{*}$ has the RNP.

COROLlary 3.3. Let $\widetilde{L}^{\infty}(B)=\left\{T \in \mathscr{D}^{\prime}(B): \widetilde{T} \in L^{\infty}(B)\right\}$.

(a) If $B^{*}$ has $R N P$ then $\operatorname{BMO}\left(B^{*}\right) \subset L^{\infty}\left(B^{*}\right)+\widetilde{L}^{\infty}\left(B^{*}\right)$.

(b) $B$ is a UMD space if and only if $\mathrm{BMO}\left(B^{*}\right)=L^{\infty}\left(B^{*}\right)+\widetilde{L}^{\infty}\left(B^{*}\right)$.

PROOF. (a) follows from Corollary 3.2 and Theorems 2.1 and 3.1. To see (b) it is a standard fact that if $L^{\infty}\left(B^{*}\right) \subset \operatorname{BMO}\left(B^{*}\right)$ then also $L^{2}\left(B^{*}\right) \subset L^{2}\left(B^{*}\right)$ which implies that $B^{*}$ and therefore $B$ are UMD spaces. On the other hand, Theorem 1.2 and the duality results give us the converse.

REMARK 3.1. S. Y. Chang and R. Fefferman [9] considered the space $H^{1}\left(\mathbf{T}^{2}\right)$ as the space of functions $f$ in $L^{1}\left(\mathbf{T}^{2}\right)$ such that

$$
\sup _{0<r<1,0<s<1} \iint P_{r}\left(t_{1}-s_{1}\right) P_{s}\left(t_{2}-s_{2}\right) f\left(s_{1}, s_{2}\right) d s_{1} d s_{2} \in L^{1}\left(\mathbf{T}^{2}\right) .
$$

We can relate $H_{\max }^{1}\left(H_{\max }^{1}\right)$ to this space by noticing that a function in $H_{\max }^{1}\left(H_{\max }^{1}\right)$ can be identified with a function $f$ in $L^{1}\left(\mathbf{T}^{2}\right)$ satisfying

$$
P^{*} f\left(t_{1}\right)=\sup _{0<r<1}\left\|\int P_{r}\left(t_{1}-s_{1}\right) f\left(s_{1}, s_{2}\right) d s_{1}\right\|_{H_{\max }^{1}} \in L^{1}(\mathbf{T}) .
$$

The proof of this identification uses a very nice observation due to $W$. Hensgen who showed that the unit ball of $H_{\max }^{1}$ is closed in $L^{1}(\mathbf{T})$ (personal communication).

With these two identifications it is very easy to verify that

$$
H_{\max }^{1}\left(\mathbf{T}^{2}\right) \subset H_{\max }^{1}\left(H_{\max }^{1}\right) .
$$

Now from duality, and since BMO fails RNP, we can get that

$$
\mathrm{BMO}(\mathrm{BMO}) \varsubsetneqq \mathrm{BMO}\left(\mathbf{T}^{2}\right) \text {, }
$$

where $\operatorname{BMO}\left(\mathbf{T}^{2}\right)$ is the space considered in [9] and which represents the dual of $H_{\max }^{1}\left(\mathbf{T}^{2}\right)$.

ACKNOWLEDGEMENTS. I would like to thank Professor Rubio de Francia for suggesting the problem and for useful conversation, and Professors D. Burkholder and J. J. Uhl for reading the manuscript and their helpful remarks and comments. 
Finally I am very grateful to $\mathrm{W}$. Hensgen who pointed out a mistake which made me change the approach in Lemma 3.3, and for all his very interesting comments and observations.

\section{REFERENCES}

1. O. Blasco, On the dual of $H_{B}^{1}$, Colloq. Math. (to appear).

2. _ Positive p-summing operators on $L_{p}$-spaces, Proc. Amer. Math. Soc. 100 (1987), 275280.

3. J. Bourgain, Some remarks on Banach spaces in which martingale difference sequences are unconditional, Ark. Mat. 21 (1983), 163-168.

4. __ Vector valued singular integrals and $H^{1}$-BMO duality, Probability Theory and Harmonic Analysis, J. A. Chao and W. A. Woyczynski, editors, Marcel Dekker, New York, 1986, pp. 1-19.

5. A. V. Bukhvalov, Duals of spaces of vector-valued analytic functions and duality of functions generated by these spaces, LOMI 92 (1979), 30-50.

6. D. L. Burkholder, A geometric condition that implies the existence of certain singular integrals of Banach-space-valued functions, Conf. Harmonic Analysis in honor of A. Zygmund, Wadsworth, Belmont, Calif., 1982, pp. 270-286.

7. __ Martingales and Fourier analysis in Banach spaces, Lecture Notes in Math., vol. 1206, Springer-Verlag, Berlin, 1986.

8. D. L. Burkholder, R. F. Gundy and M. L. Silverstein, A maximal function characterization of the class $H^{p}$, Trans. Amer. Math. Soc. 157 (1971), 137-153.

9. S.-Y. A. Chang and R. Fefferman, Some recent developments in Fourier analysis and $H^{p}$-theory in product domains, Bull. Amer. Math. Soc. (N.S.) 12 (1985), 1-43.

10. R. R. Coifman, A real variable characterization of $H^{p}$, Studia Math. 51 (1974), 269-274.

11. R. R. Coifman and G. Weiss, Extension of Hardy spaces and their use in analysis, Bull. Amer. Math. Soc. 83 (1977) 569-645.

12. J. Diestel and J. J. Uhl, Vector measures, Math. Surveys, no. 15, Amer. Math. Soc., Providence, R.I., 1977.

13. N. Dinculeanu, Linear operators on $L^{p}$-spaces. Vector and operator valued measures and applications, Proc. Sympos. Snowbird Resort (Alta, Utah), Academic Press, New York, 1972, pp. 109-124.

14. P. L. Duren, Theory of $H^{p}$-spaces, Academic Press, New York, 1970.

15. C. Fefferman, Characterization of bounded mean oscillation, Bull. Amer. Math. Soc. 77 (1971), 587-588.

16. C. Fefferman and E. M. Stein, $H^{p}$-spaces of several variables, Acta Math. 129 (1972), 137-193.

17. J. Garcia-Cuerva and J. L. Rubio de Francia, Weighted norm inequalities and related topics, North-Holland, Amsterdam, 1985.

18. W. Hensgen, Hardy-Raume vektorwertiger Funktionen, Thesis, München 1986.

19. F. John and L. Nirenberg, On functions of bounded mean oscillation, Comm. Pure Appl. Math. 14 (1961), 415-426.

20. J. L. Journé, Calderón-Zygmund operators, pseudo-differential operators and the Cauchy integral of Calderón, Lecture Notes in Math., vol. 994, Springer-Verlag, Berlin, 1983.

21. Y. Katnelson, An introduction to harmonic analysis, Wiley, New York, 1968.

22. H. E. Lacey, The isometric theory of classical Banach spaces, Springer-Verlag, Berlin, 1974.

23. J. L. Rubio de Francia, Fourier series and Hilbert transforms with values in UMD Banach spaces, Studia Math. 81 (1985), 95-105.

24. J. L. Rubio de Francia and J. L. Torrea, Some Banach techniques in vector valued Fourier analysis, Colloq. Math. (to appear).

25. F. Ruiz and J. L. Torrea, Sobre el dual de espacios de Hardy de funciones con valores vectoriales, Proc. 8th Portuguese-Spanish Conf. Math., vol. I, Univ. of Coimbra, 1981, pp. 257-261.

26. H. H. Schaefer, Banach lattices and positive operators, Springer-Verlag, Berlin, 1974.

Departmento de Matematicas, Universidad de Zaragoza, Zaragoza-50009, SPAIN 